\title{
Charge distribution in soft chiral pion bremsstrahlung.
}

\author{
I.V. Andreev ${ }^{1}$, M. Biyajima ${ }^{2}$, V.A. Nechitailo ${ }^{1}$, N. Suzuki ${ }^{3}$ \\ ${ }^{1}$ P.N. Lebedev Physical Institute, Moscow, Russia \\ ${ }^{2}$ Department of Physics, Faculty of Liberal Arts, \\ Shinshu University, Matsumoto 390, Japan \\ ${ }^{3}$ Matsusho Gakuen Junior College, Matsumoto 390-12, Japan
}

\begin{abstract}
Bremsstrahlung of soft chiral pions in high-energy processes is considered. The distribution over number of neutral and charged pions is shown to obey the inverse square root law. This law is argued to be generic for multiple coherent production of soft chiral pions.
\end{abstract}

\section{Introduction}

Charge distribution of pions in multiple production processes drew much attention recently. The growth of interest in this subject is due to expectations to detect the disoriented chiral condensate (DCC) formation in high energy collisions [1]-[6]. The simplest picture of the process is given by "Baked Alaska scenario" [- 1 , where coherent pulses of semiclassical pion field are emitted leading to anomalously large fluctuations in the ratio of neutral to charge pions produced. In particular, the probability to produce $n_{o}$ neutral pions (for large total number of pions $n$ ) is given by inverse square root formula,

$$
w\left(n_{0}\right) \sim 1 / \sqrt{n_{0} n},
$$

being very flat and so quite different from usual binomial-like distributions. This mechanism may be relevant for description of "Centauro" (and "Anti-Centauro") type events found in cosmic ray experiments, see [7, 8] and references therein, in which the number of charged particles drastically exceeds the number of neutral ones (or vice versa).

Now the problem arises - to what extent the behaviour in Eq. (1) can be considered as a signature of DCC formation. Let us remind in this connection that the distribution of the form of Eq. (1) was found long ago in a model of independent coherent pion production when isotopic spin conservation was taken into account [9, 10]. In these and previous works [11, 12] the coherent production of pions was taken for granted.More recently the topic of coherent and squeezed states was discussed in the literature in the context of DCC [13]-[18]. It was found that charge distribution in squeezed states,having small isotopic spin, is also very broad [18]. 
In the present paper we consider a concrete mechanism of pion production - soft chiral pion bremsstrahlung accompanying some basic high-energy process and estimate charge distribution of the pions. The quantum charge states of chiral pions emitted from simple vertices will be explicitly calculated. It will be found that neutral pion number distribution again has the form of Eq. (11). That is, such flat charge distributions are typical for soft chiral pions and do not indicate directly on DCC formation.

The soft chiral pion bremsstrahlung was studied many years ago [19, 20]. Similarly to photons, soft pions are emitted from external lines of diagrams representing the basic process (to be definite, we shall take external particles to be spin $1 / 2$ fermions (nucleons)). The complications arising due to noncommutative pion-nucleon vertices and nonlinear pion-nucleon coupling were shown to be mutually cancelled [19. Nonlinear pion-pion coupling can be taken into account but its effect vanishes in the limit of small pion momenta. Soft virtual pion exchange [21] changes normalization and does not influence the pion number distributions. The net result for soft pion emission is given by substitution [20]:

$$
\psi_{j} \rightarrow \exp \left(-i \gamma_{5} \tau_{i} \phi_{i} / 2\right) \psi_{j}
$$

where $\psi_{j}$ is the fermion field for every incoming or outgoing nucleon in the skeleton diagram of a basic process, $\phi_{i}=\pi_{i} / f_{\pi}, \pi_{i}$ being the pion field, $f_{\pi}=93 \mathrm{MeV}$ is the pion decay constant. The substitution in Eq. (2) can be deduced [20, 22] from requirement for the effective lagrangian (S-matrix) of strongly interacting fermions with accompanying soft pion emission to be chiral invariant if it is isotopic invariant without these additional pions.

To simplify calculations we shall consider here the skeleton vertex for two fermions, that is $\bar{\psi} \Gamma \psi$, where $\Gamma$ may contain Dirac matrices $\gamma_{\mu}$ and (or) isotopic Pauli matrices $\tau_{i}$. In the absence of isotopic matrices the vector $\left(\Gamma=\gamma_{\mu}\right)$ and axial $\left(\Gamma=\gamma_{\mu} \gamma_{5}\right)$ vertices are chiral invariant and do not produce the additional soft pions we are considering here. Other vertices do allow the emission of pions.

\section{Scalar vertex}

As the simplest example consider the scalar vertex $\bar{\psi} \psi$ ( $\Gamma$ is the identity matrix). Its chiral-invariant extension has the form

$$
V_{s}=\bar{\psi} \exp \left(-i \gamma_{5} \tau_{i} \phi_{i}\right) \psi(x) .
$$

It coincides formally with the modified nucleon mass term in the chiral lagrangian. We neglect pion momenta and for the fields $\phi_{i}$ use the decomposition

$$
\phi_{i} \rightarrow \phi_{i}(0)=\phi_{i}^{+}+\phi_{i}^{-}=\int d^{3} k f(k)\left[a_{i}^{+}(k)+a_{i}^{-}(k)\right]
$$


where creation and annihilation operators $a_{i}^{+}(k), a_{i}^{-}(k)$ obey canonical commutation relations. In the free field approximation

$$
f(k)=(2 \pi)^{-3 / 2}\left(2 k_{0}\right)^{-1 / 2} f_{\pi}^{-1}, \quad k_{0} \leq k_{m}
$$

where $k_{m}$ is an upper limit of pion softness. The most severe estimation for this limiting momentum is the rho-meson mass ensuring applicability of the chiral lagrangian technique though it may well appear to be higher.In any case it does not exceed the momentum transfer $\Delta p$ in the vertex $\Gamma, k_{m}<\Delta p$. To calculate the matrix elements of $\pi^{0}, \pi^{+}, \pi^{-}$production

$$
M_{s}=\left\langle n_{+}, n_{-}, n_{0}\left|\exp \left(-i \gamma_{5} \tau_{i} \phi_{i}(0)\right)\right| 0\right\rangle
$$

it appears convenient to use the integral representation

$$
\exp \left(-i \gamma_{5} \tau_{i} \phi_{i}\right)=\frac{1}{4 \pi} \int d \Omega e^{i e_{k} \phi_{k}}\left(1+i e_{k} \phi_{k}-i \gamma_{5} \tau_{k} \phi_{k}\right)
$$

where $\vec{e}$ is the unit vector in three dimensions and integration is performed over solid angles of the vector $\vec{e}$.

The total isotopic spin of the pions produced can be zero or one. Consider the first case and decompose the pion fields into creation and annihilation parts, $\phi=\phi^{+}+\phi^{-}$. Using equations

$$
\begin{array}{r}
{\left[\phi_{i}^{-}, \phi_{i}^{+}\right]=c \delta_{i j}, \quad e^{i e_{k} \phi_{k}}|0\rangle=e^{-c / 2} e^{i e_{k} \phi_{k}^{+}}|0\rangle} \\
c=\int d^{3} k|f(k)|^{2}
\end{array}
$$

we are led to consider a pion state of the form:

$$
\left|\Phi_{s}\right\rangle=\left(1+\frac{d}{d \alpha}\right)_{\alpha=1} \frac{1}{4 \pi} \int d \Omega e^{-\alpha^{2} c / 2+i \alpha e_{k} \phi_{k}^{+}}|0\rangle .
$$

The normalization factor $N_{s}$ of the state is

$$
N_{s}=\left\langle\Phi_{s} \mid \Phi_{s}\right\rangle=\frac{1}{2}\left[1-(4 c-1) e^{-2 c}\right]
$$

and the average number of pions produced is

$$
\langle n\rangle=\frac{1}{N_{s}}\left\langle\Phi_{s}\left|\int d^{3} k a_{i}^{+}(k) a_{i}^{-}(k)\right| \Phi_{s}\right\rangle=c \frac{1+(4 c-1) e^{-2 c}}{1-(4 c-1) e^{-2 c}} .
$$

If the average number of pions is large (it is the most interesting case) then

$$
\langle n\rangle \cong c, \quad c \gg 1
$$

To estimate it take the free field approximation (5); then 


$$
\langle n\rangle=\frac{1}{f_{\pi}^{2}} \int \frac{d^{3} k}{(2 \pi)^{3} 2 k_{0}} \cong \frac{k_{m}^{2}}{8 \pi^{2} f_{\pi}^{2}}
$$

that is, to produce many soft pions by the present mechanism one needs momentum transfer in the vertex to be large enough

$$
\Delta p>k_{m}>2 \pi f_{\pi}^{2} \cong 0.6 \mathrm{GeV}
$$

A prominent feature of the model is the distribution over number of charged and neutral pions produced. It can be obtained from matrix elements (6) and has the multiplicative form with respect to the total number of pions $n=n_{0}+n_{c}$ and the number of neutral pions $n_{0}$,

$$
w\left(n, n_{0}\right)=w(n) w_{n}\left(n_{0}\right)
$$

where

$$
w_{n}\left(n_{0}\right)=\frac{1}{n+1} \frac{(n / 2) !}{\Gamma\left(\frac{n+1}{2}\right)} \frac{\Gamma\left(\frac{n_{0}+1}{2}\right)}{\left(n_{0} / 2\right) !} \approx \frac{1}{\sqrt{n n_{0}}}
$$

is the probability to produce $n_{0}$ neutral pions for the given total number of pions $\left(\Gamma(n)\right.$ is the Euler $\Gamma$-function, $n_{0}$ and $n$ are even, $\left.n_{0} \leq n\right)$, and

$$
w(n)=\frac{(n-c+1)^{2}}{N_{s}} \frac{e^{-c} c^{n}}{(n+1) !}
$$

is the distribution over the total number of pions produced.

The distribution (16) over the number of neutral pions $n_{0}$ and corresponding distribution over the number of charged pions

$$
w_{n}\left(n-n_{c}\right) \approx \frac{1}{\sqrt{n\left(n-n_{c}\right)}}
$$

are very broad. This means that the probabilities for events to have almost all pions being charged or neutral are not negligible even for $n \gg 1$. These distributions appear to be very similar to those invented some time ago [9, 10] for the explanation of Centauro-type events. The distribution (17) over the total number of pions is Poisson-like (though with an additional central dip at $n \sim\langle n\rangle$ ) and it is much more narrow than (16), (18).

\section{Electromagnetic vertex}

As a case of immediate physical interest consider the soft pion emission for electromagnetic scattering of strongly interaction fermions. The skeleton vertex has now the form 


$$
V_{0}=e \bar{\psi} \gamma_{\mu} Q \psi=e \bar{\psi} \gamma_{\mu} \frac{\tau_{3}+N_{B}}{2} \psi
$$

where the baryon number $N_{B}=1$ for nucleons and $Q$ is electric charge in units of $e$. Chiral extension of the vertex is taken as

$$
V=e \bar{\psi} e^{-i \gamma_{5} \tau_{k} \phi_{k} / 2} \gamma_{\mu} \frac{\tau_{3}+N_{B}}{2} e^{-i \gamma_{5} \tau_{k} \phi_{k} / 2} \psi
$$

leading to the substitution

$$
\gamma_{\mu} \tau_{3} \rightarrow \gamma_{\mu} \tau_{3}+\gamma_{\mu} \gamma_{5} \varepsilon_{i j 3} \phi_{i} \tau_{j} \frac{\sin \phi}{\phi}-\gamma_{\mu}\left(\tau_{3} \phi^{2}-\phi_{3} \tau_{k} \phi_{k}\right) \frac{1-\cos \phi}{\phi^{2}}, \quad \phi=\sqrt{\phi_{k}^{2}}
$$

We consider here diagonal transitions. Then the pion state has the form

$$
\begin{aligned}
\left|\Phi_{e}\right\rangle & =\left(\phi_{1}^{2}+\phi_{2}^{2}\right)(1-\cos \phi) / \phi^{2}|0\rangle= \\
& =\frac{1}{4 \pi} \int_{|\vec{x}| \leq 1} \frac{d^{3} x}{|\vec{x}|}\left(-\frac{\partial^{2}}{\partial x_{1}^{2}}-\frac{\partial^{2}}{\partial x_{2}^{2}}\right) e^{i x_{k} \phi_{k}}|0\rangle
\end{aligned}
$$

and its isotopic spin is equal to zero. The normalization factor is

$$
\begin{array}{r}
N_{e}=\left\langle\Phi_{e} \mid \Phi_{e}\right\rangle=\frac{4}{5}+\frac{16}{15}(c-1) e^{-c / 2}-\frac{4}{15}(4 c-1) e^{-2 c} \\
N_{e}=\left\{\begin{array}{l}
2 c^{2} \text { for } c \ll 1 \\
4 / 5 \text { for } c \gg 1
\end{array}\right.
\end{array}
$$

where $c$ is given by Eq. (8) and the average number of pions is

$$
\begin{array}{r}
\langle n\rangle=\frac{4}{15 N_{e}}\left[c+3-4 e^{-c / 2}+\left(4 c^{2}-c+1\right) e^{-2 c}\right] \\
\langle n\rangle= \begin{cases}1 & \text { for } c \ll 1 \\
\frac{1}{3} c & \text { for } c \gg 1\end{cases}
\end{array}
$$

As in the previous case, the most interesting characteristic is the distribution over the number of pions of different charges. Considering the state (22) one has to calculate the matrix elements

$$
\begin{aligned}
M_{e} & =N_{e}^{-1 / 2}\left\langle n_{+}, n_{-}, n_{0} \mid \Phi_{e}\right\rangle= \\
& =N_{e}^{-1 / 2} \frac{1}{4 \pi} \int_{|\vec{x}| \leq 1} \frac{d^{3} x}{|\vec{x}|}\left(-\frac{\partial^{2}}{\partial x_{1}^{2}}-\frac{\partial^{2}}{\partial x_{2}^{2}}\right)\left\langle 0\left|a_{i_{1}}\left(q_{1}\right) \ldots a_{i_{n}}\left(q_{n}\right) e^{i x_{k} \phi_{k}}\right| 0\right\rangle \\
& =K \int \frac{d^{3} x}{2 \pi|\vec{x}|} \frac{\partial}{\partial x_{+}} \frac{\partial}{\partial x_{-}}\left[e^{-c x^{2} / 2} x_{+}^{n_{+}} x_{-}^{n_{-}} x_{3}^{n_{0}}\right]
\end{aligned}
$$


where

$$
K=\frac{-i^{n}}{N_{e}^{1 / 2}} \prod_{j=1}^{n} f\left(q_{j}\right), \quad \prod_{j} \int d q_{j}|K|^{2}=\frac{c^{n}}{N_{e}^{1 / 2}}, \quad x_{ \pm}=\frac{1}{\sqrt{2}}\left(x_{1} \pm i x_{2}\right) .
$$

The matrix element for transition to the state, which contains $n_{0}$ neutral pions and $n_{c}$ charged pions can be represented as the sum of two terms:

$$
\begin{array}{r}
M_{e}=\frac{K}{c^{n / 2}} \frac{2^{n_{0} / 2} \Gamma\left(\frac{n_{0}+1}{2}\right)\left(\frac{n_{c}}{2}\right) !}{\Gamma\left(\frac{n+3}{2}\right)} \\
{\left[\frac{\left(n-3 n_{0}\right)}{2 n(n+3)} \gamma\left(\frac{n}{2}+1, \frac{c}{2}\right)+\left(\frac{n_{c}(n+1)}{2 n}-\frac{c\left(n_{c}+2\right)}{2(n+3)}\right)\left(\frac{c}{2}\right)^{n / 2} e^{-c / 2}\right]}
\end{array}
$$

where $n_{c}$ and $n_{0}$ are even, $n=n_{0}+n_{c}, n \neq 0$ and

$$
\gamma\left(\frac{n}{2}+1, \frac{c}{2}\right)=\int_{0}^{c / 2} d u u^{n / 2} e^{-u}
$$

Consider the most interesting situation, when the average number of pions in the final state in Eq. (26) is large, $c \gg 1$. Then the first term in square brackets in Eq. (28) dominates for small numbers of pions, $n \sim 1$, and the second term dominates for large $n \sim c$, their interference being small. Therefore the second term gives the distribution over the number of neutral and charged pions in high multiplicity events. It reads:

$$
\begin{array}{r}
w_{2}\left(n, n_{0}\right) \cong \frac{3\left(c-n_{0}\right)^{2}}{2 N_{e} c^{2} \sqrt{2 c}} \frac{\Gamma\left(\frac{n_{0}+1}{2}\right)}{\left(\frac{n_{0}}{2}\right) !} w_{2}(n) \\
w_{2}(n) \cong \frac{2}{3 c \sqrt{2 \pi c}}(n-c)^{2} \exp \left(-\frac{(n-c)^{2}}{2 c}\right)
\end{array}
$$

where $w_{2}(n)$ is the probability to find $n=n_{0}+n_{c}$ pions, $n$ and $n_{0} \leq n$ are even, $N_{e} \cong 4 / 5$.

The distribution over the number of neutral (or charged) pions in Eq. (29) is again very broad ensuring a sizable number of events, in which almost all pions are neutral (or charged). The distribution $w_{2}(n)$ over the total number of pions is again narrow and in fact coincides with Eq. (17) for $c \gg 1, n \gg 1$ up to a normalization factor. The total probability of high multiplicity events is

$$
\sum_{n=2 k} w_{2}(n)=\frac{1}{3}
$$

just corresponding to factor $1 / 3$ in Eq. (25). 
Small multiplicity events are given by the first term in square brackets in Eq. (28). For large average multiplicities $c \gg 1$ the corresponding probability is

$$
\begin{array}{r}
w_{1}\left(n \neq 0, n_{0}\right) \cong \frac{1}{N_{e}} \frac{\sqrt{\pi} \Gamma\left(\frac{n_{0}+1}{2}\right)}{\left(\frac{n_{0}}{2}\right) !} \frac{\left(n-3 n_{0}\right)^{2}}{4 n^{2}(n+3)^{2}} \frac{\Gamma^{2}\left(\frac{n}{2}+1\right)}{\Gamma^{2}\left(\frac{n}{2}+\frac{3}{2}\right)} \\
w_{1}(0,0) \cong 5 / 9
\end{array}
$$

where $n$ and $n_{0}$ are even and $n_{0} \leq n$. Performing the sum over the number of neutral pions in Eq. (32) we obtain the distribution over the total number of pions in low multiplicity events:

$$
\begin{aligned}
w_{1}(n) & =\frac{1}{2 n(n+3)} \frac{\sqrt{\pi} \Gamma\left(\frac{n}{2}+1\right)}{\Gamma\left(\frac{n}{2}+\frac{3}{2}\right)}, \quad n \neq 0, c \gg 1 \\
\sum_{n=2 k \neq 0} w_{1}(n) & =\frac{1}{9}
\end{aligned}
$$

According to Eq. (34), the average number of $\pi$ pairs in low multiplicity events is very small, equal to $1 / 3$ on the average,

$$
\sum_{n=2 k} \frac{n}{2} w_{1}(n)=\frac{1}{3} .
$$

\section{Discussion and conclusions}

Two examples of soft chiral pion emission considered above show very broad distributions over the number of charged and neutral pions in high multiplicity

events. The neutral pion distributions in both cases are given essentially by the function

$$
\begin{aligned}
& w\left(n_{0}\right) \cong \frac{1}{\sqrt{2\langle n\rangle}} \frac{\Gamma\left(\frac{n_{0}}{2}+\frac{1}{2}\right)}{\Gamma\left(\frac{n_{0}}{2}+1\right)}, \quad\left(n_{0} \text { even }\right) \\
& w\left(n_{0}\right) \cong \frac{1}{\sqrt{\langle n\rangle n_{0}}} \quad \text { for } n_{0} \gg 1, n_{0} \text { even }
\end{aligned}
$$

which is similar to Eq. (1). This distribution gives the sizable probability to find events with a small number of neutral pions. For example, the probability to find no neutral pions is

$$
w(0)=\sqrt{\pi / 2\langle n\rangle}
$$


amounting here to more than $10 \%$ for $\langle n\rangle=100$. As the distribution over the total number of pions $n$ is rather narrow for $\langle n\rangle \gg 1$, the charged pion distribution is given mainly by the same function with substitution $n_{0}=n-n_{c}$.

The conditions for such broad distributions to appear in high multiplicity events are small isotopic spins of the pion system and many particle matrix elements symmetric with respect to pion momenta, thus ensuring a constructive interference. In other words, the pion emission must be coherent円. This can be seen already from an early paper by A. Pais [15] and was explicitly demonstrated more recently in paper [16]. Both of these conditions are fulfilled in our chiral model examples.

The bremsstrahlung spectrum of pseudoscalar pions has the form $d n \sim k d k$ (contrary to photon spectrum $d k / k$ ) and so very small momenta $k$ are inefficient for this mechanism.It was necessary (as in Bloch-Nordsiek model) to introduce an upper limit of pion softness, $k<k_{m}$ and the total number of pions produced by this mechanism is proportional to $k_{m}^{2}$. The value of $k_{m}$ is not quite definite (the most severe possible estimation is around rho-meson mass) but it does not exceed the momentum transfer $\Delta p$ in the baryonic vertex $\Gamma$.Anyhow it is clear that the presence of large baryonic momentum transfer $\Delta p$ (and so the presence of high $p_{T}$ baryons) is highly favourable for copious production of pions by the present mechanism. At the same time the soft pions are expected to be present in lower $p_{T}$ region.In the last region the pion spectrum of the form $k d k$ by itself can be used for identification of the process of pion bremsstrahlung.It can be seen in future experiments when it will be possible to look at narrow windows of $p_{T}$.

One can attempt to apply this mechanism for a description of "Centauro" and "Anti-Centauro" events in cosmic rays. The average transverse momentum of particles in these events is just very high, three to six times the value typical for hadronic processes, see Ref. [8], where the compilation of exotic events in cosmic rays is given.

In conclusion, it thus appears that inverse square root distributions over number of neutral and charged pions are of very general nature being characteristic for coherent soft pion radiation.

\section{Acknowledgments}

This work was supported in part by the JSPS Program on Japan-FSU Scientists Collaboration. I.A. and V.N. were also supported by Russian Fund for Fundamental Research,grant 96-02-16210a.

\section{References}

\footnotetext{
${ }^{1}$ In its simplest phenomenological version the corresponding pion state is the eigenstate of the annihilation operator of isoscalar pion pair and it can be considered as a coherent state for an isoscalar pair, see (10])
} 
[1] A.A. Anselm and M.G. Ryskin. Phys.Lett.B266 (1991) 482.

[2] J.-P. Blaizot, A. Krzywicki. Phys.Rev. D46 (1992) 246.

[3] J. Bjorken. Int.J.Mod.Phys.A7 (1992) 4189; Acta Phys. Pol B23 (1992) 561.

[4] J. Bjorken, K. Kowalsky and C. Taylor. Proc. of the 7th Recontres de Physique de la Valle d'Aoste, La Thoile, Italy, 1993.

[5] K. Rajagopal and F. Wilczek. Nucl.Phys.B399 (1993), 395; B404 (1993) 577.

[6] Z. Huang and X.N. Wang. Phys. Rev. D49 (1994) 4339.

[7] C.M.G. Lattes, Y. Fujimoto, S. Hasegawa. Phys.Rep.65 (1980) 151.

[8] A.D. Panagiotou, A. Karabarbounis, A. Petridis. Z.Phys.A Atomic Nuclei 333 (1989) 536.

[9] I.V. Andreev. JETP Lett. 33 (1981) 367.

[10] I.V. Andreev. Proc. of the XVII Rec. de Moriond, Les Arc (1982).

[11] D. Horn and R. Silver. Ann.Phys.66 (1971) 59.

[12] J.C.Botke,D.J.Scalapino and R.L.Sugar. Phys.Rev.D9(1974) 813.

[13] Ian I.Kogan. JETP Lett.59(1994) 307.

[14] Thomas D.Cohen,Manoj K.Banergee,Marina Nielsen and Xuemin Jin. Phys.Lett.333(1994) 166.

[15] R.D.Amado and Ian I.Kogan. Phys.Rev.D51(1995) 190

[16] Y.Kluger,F.Cooooper,E.Mottola,J.P.Paz and A.Korner. Nucl.Phys. A590 (1995) 581c.

[17] M.Martinis,V.Mikita-Martinis,A. $\breve{S}$ varc,J.C̆ ruugelj. Fizika B3(1994) 197.

[18] I.M.Dremin and R.C.Hwa.Phys.Rev.D59(1996) 1216.

[19] S.Weinberg. Phys.Rev.D2 (1970) 674.

[20] L.S.Brown.Phys.Rev.D2 (1970) 3083.

[21] V.Ya.Fainberg and R.E.Kallosh. P.N.Lebedev Inst. Preprint N170 (1970).

[22] S.Coleman, J.W.Wess and B.Zumino. Phys.Rev. 177 (1968) 2239.

[23] A.Pais. Ann. of Phys.9 (1960) 548.

[24] V.A.Karmanov, A.E.Kudriavtzev. Preprint ITEP-88 (1983). 